Reprod. Nutr. Dévelop., 1986, 26 (1 B), 315-316.

\title{
Evolution nycthémérale des taux d'acides gras volatils dans les contenus digestifs du lapin
}

T. GIDENNE

Laboratoire de Recherches sur l'Elevage du Lapin,

I.N.R.A., Chemin de Borde Rouge, B.P. 27,

Auzeville, 31326 Castanet-Tolosan Cedex.

Summary. After slaughter of young rabbits every $3 \mathrm{~h}$, total free fatty acid (FFA) concentration was measured by CPG in four digesta (stomach, ileum, caecum and rectum). FFA levels, maximal in the caecum and rectum (50 to $100 \mathrm{mM} / \mathrm{l})$, were related to the practice of caecotrophy.

Introduction. Le lapin diggère partiellement les constituants des parois végétales, essentiellement par sa flore cæcale. Nous avons suivi l'évolution nycthémérale quantitative et qualitative des acides gras volatils (dosés par chromatographie en phase gazeuse) de digesta prélevés à 4 niveaux : estomac, iléon, cæcum, rectum.

Matériel et méthodes. Trois aliments à teneurs identiques en matières azotées $(14,5 \%)$, en énergie (2340 kcal ED/ kg MS) et en cellulose Van Soest $(23 \%)$, mais à teneurs différentes en lignine Van Soest $(6,3 ; 10,0 ; 13,7)$ sont distribués, ad libitum, à 3 lots de 36 lapins Néozélandais Blancs âgés de 9 semaines. Les digesta sont prélevés après abattage, toutes les $3 \mathrm{~h}$, durant $24 \mathrm{~h}$. Leur composition a déjà été décrite (Gidenne et Lebas, 1984). L'absence d'interactions entre les effets " régime " et " heure de prélèvement ", permet de grouper les résultats obtenus avec 3 aliments.

Résultats et discussion. Bien que faible, la teneur en AGV totaux varie significativement au cours de la journée dans l'estomac (fig. 1) ; mais varie peu au niveau iléal. Très élevée dans le cæacum, cette teneur baisse de $25 \%$ entre $9 \mathrm{~h}$ et $15 \mathrm{~h}$, puis progresse pour atteindre son maximum entre $21 \mathrm{~h}$ et $24 \mathrm{~h}$. Dans le rectum, elle augmente brusquement de $35 \%$ entre $6 \mathrm{~h}$ et $9 \mathrm{~h}$; le matériel digestif est alors composé essentiellement de cæcotrophes riches en AGV. Cette teneur baisse ensuite progressivement jusqu'à $21 \mathrm{~h}$, ce qui correspond à la présence des crottes dures. Cette liaison entre cæcotrophie et production d'AGV s'observe aussi au niveau cæcal et stomacal. La vidange du cæcum, qui précède l'émission des cæcotrophes, entraîne une baisse de sa teneur en AGV totaux. Puis l'arrivée de nouveaux nutriments semble stimuler l'activité fermentaire; ceci se traduit par un accroissement de $46 \%$ de la concentration en AGV totaux entre $15 \mathrm{~h}$ et $24 \mathrm{~h}$. Au niveau stomacal, l'acidité volatile totale augmente lors de l'ingestion des cæcotrophes entre $9 \mathrm{~h}$ et $12 \mathrm{~h}$. Par ailleurs, à $3 \mathrm{~h}$, certains animaux pratiquent la cæcotrophie ce qui entraîne une modification de la teneur en AGV totaux au niveau stomacal, cæecal et rectal. Parker (1976) n'observe pas de telles variations au 
cours de la journée ; il conclut à l'absence de relation entre cacotrophie et production d'AGV. Par contre, Le Bars et al. (1971) au niveau cacal et Vernay (1975) au niveau du côlon, signalent des variations semblables aux nôtres. Dans le mélange d'AGV de l'estomac et de l'iléon, les proportions moyennes d'acétate (C2) sont élevées (respectivement 86,8 et $84,1 \%$ ), ainsi que celles du valérate (C5) $(4,3$ et $6,8 \%)$, ceci aux dépens du propionate (C3) $(1,6$ et $0,2 \%)$ et du butyrate (C4) $(6,0$ et $6,8 \%)$. Par contre, au niveau cæcal et rectal, les proportions moyennes de $\mathrm{C} 3(4,6$ et $4,9 \%)$ et de $\mathrm{C} 4(14,9$ et $9,0 \%)$ sont alors plus fortes essentiellement aux dépens de $\mathrm{C} 5(0,9$ et $1,7 \%)$, mais aussi de $\mathrm{C} 2(78,4$ et $83,3 \%)$. Au cours du nycthémère, les proportions molaire de chaque AGV varient peu au niveau iléal et cæcal. Par contre, la proportion de $\mathrm{C} 2$ est plus faible dans les cæcotrophes que pour les crottes dures et inversement dans le cas de $\mathrm{C} 4$; ce qui détermine des variations plus marquées au niveau rectal et stomacal.

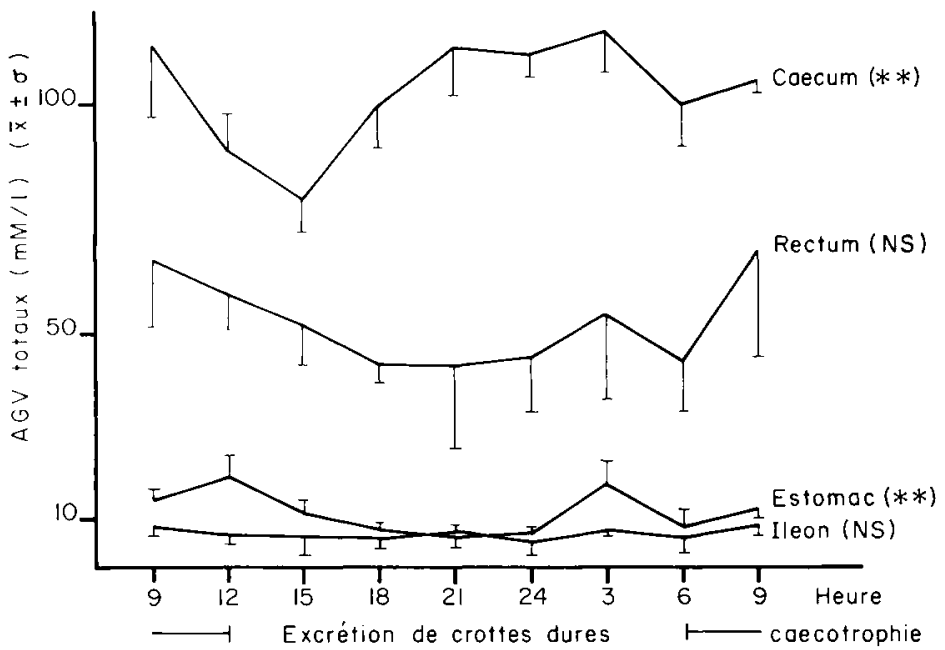

FIG. 1. - Evolution du taux d'AGV totaux en fonction de l'heure et du lieu de prélèvement.

En conclusion, la teneur en AGV cacale et rectale chez le lapin est importante et comparable à celle du rumen, mais la composition molaire est différente (CA > C3). Ceci s'explique par les particularités digestives du lapin, tant au niveau de la flore cacale, qu'au niveau du cycle digestif du lapin qui semble lié à l'évolution circadienne des taux d'AGV.

Gidenne T., Lebas F., 1984. Evolution circadienne du contenu digestif chez le lapin en croissance. Relation avec la cæcotrophie. Congr. mond. Cunicult., Avril 1984, Rome.

Le Bars H., Guémon L., Demaux G., 1971. Production d'acides gras volatils dans le cæcum du lapin. Ann. Biol. anim. Bioch. Biophys., 11, 301-302 (Abstr.).

Vernay M., Raynaud P., 1975. Répartition des acides gras volatils dans le tube digestif du lapin domestique. I) Lapins alimentés en luzerne et avoine. Ann. Rech. vét., 6, 357-368.

Parker D. S., Mc Millian R. T., 1976. The determination of volatile fatty acids in the caecum of the conscious rabbit. Br. J. Nutr., 35, 365 . 